\title{
SOME ASPECTS OF ION-ACOUSTIC WHISTLERS IN THE IONOSPHERE IN THE PRESENCE OF NEGATIVE IONS
}

\author{
A.K. SUR, G.C. DAS," B. CHAKRABORTY, S.N. PAUL ** \\ Plasma Physics Group \\ Department of Mathematics \\ Jadavpur University \\ Calcutta - 700032 \\ INDIA \\ and

\section{DEBNATH} \\ Departinent of Mathematics \\ University of Central Florida \\ Orlando, Florida 32816 U.S.A. \\ (Received November 16, 1986 and in revised form July 20, 1988)
}

ARSTRACT. A study is made of the propagation of ion-acoustic whistlers in the atmosphere including the effects of negative ions. The dispersion relation, phase and group velocities of whistlers are discussed. It is shown that the presence of negative ions introduces a critical frequency which, for equal ionic masses, is equal to the ion-cyclotron frequency. Special attention is given to the group travel time of whistlers at mid-latitude and equator so that the role of negative ions on the group travel time can be determined. The cyclotron damping of whistlers in the presence of negative ions has been studied. The velocity distribution, total attenuation and the induced magnetic field are calculated from the temporal as well as spatial cyclotron damping. It is suggested that the attenuation of whistlers may cause heating of the ionosphere. It is also indicated that the measurement of the group travel time from its source to the observer at the satellite would help to diagnose the ionospheric parameters. The results of the analysis are presented by several graphical presentations.

KEY WORDS AND PHRASES. Ion-acoustic whistlers, group travel time and critical frequency.

1980 AMS SUBJECT CLASSIFICATION CODE.

1. INTRODUCTION

Considerable studies have been made on the propagation and generation of ioncyclotron waves in multicomponent plasmas and in the ionosphere. Gurnett and his associates [1-2] have made an investigation of proton whistlers and discussed the use of whistlers for diagnostic purposes in the ionosphere. Several authors including Das and Uberoi [3-4] have also considered the role of negative ions on low-frequency waves in different problems in the ionosphere and in the laboratory plasmas. 
In spite of these investigations, various aspects of ion-acoustic whistlers in the ionosphere in the presence of negative ions have not yet receivec full attention. The main purpose of this paper is to consider a plasma model that includes the effect of negative ions, and to calculate the group travel time for the whistlers observed at the equator and at the mid-latitude. In this study, we assume that the ratio of the total number of negative ions to the positive ions is less than unity, and it varies from 0.0 to 0.5 in the ionosphere. The dispersion relation, phase and group velocities of whistlers are discussed in some detail. It is shown that the presence of negative ions introduces a critical frequency which, for equal ionic masses, is equal to the ion-cyclotron frequency. Special attention is given to the group travel time of whistlers at mid-latitude and the equator. Special attention is given to the cyclotron damping of whistlers in the presence of negative ions. The velocity distribution, total attenuation and the induced magnetic field are calculated from the temporal as well as spatial cyclotron damping. This study reveals that the attenuation of whistlers may cause heating of the ionosphere. The measurement of the group travel time from its source to the observer at the satellite would help to diagnose the ionospheric parameters. Results of this analysis are presented by sevéral graphical presentations.

\section{ASSUMPTIONS AND BASIC EQUATIONS}

We assume that the ionosphere consists of several species of positive ions (subscript ' $i$ ') having mass $m_{i}$; velocity $v_{i}$ and number density $n_{i}$ and the negative ions (subscript ' $j$ ') of mass $m_{j}$; velocity $v_{j}$; and number density $n_{j}$. The corresponding quantities for electrons (subscript ' $e^{\prime}$ ) are, $m_{e}, v_{e}$ and $n_{e}$. The basic system of equations governing the dynamics of ionospheric plasma are given in usual notations (Stix [7]):

$$
\begin{gathered}
\frac{\partial n_{\alpha}}{\partial t}+\operatorname{div}\left(n_{\alpha} \underline{v}_{-\alpha}\right)=0 \\
\frac{\partial \underline{v}}{\partial t}+\left(\underline{v}_{-\alpha} \cdot \nabla\right) \underline{v}_{\alpha}=\frac{q_{\alpha}}{m_{\alpha}}\left[\underline{E}+\frac{1}{c}\left(\underline{v}_{\alpha} \times \underline{H}\right)\right] \\
\nabla \times \underline{H}=\frac{1}{c} \frac{\partial \underline{E}}{\partial t}+\frac{4 \pi \underline{j}}{c} \\
\nabla \times \underline{E}=-\frac{1}{c} \frac{\partial \underline{H}}{\partial t} \\
\nabla \cdot \underline{H}=0 \\
\nabla \cdot \underline{E}=4 \pi \Sigma n_{\alpha} q_{\alpha}
\end{gathered}
$$

where $\underline{\mathrm{J}}=\Sigma n_{\alpha} q_{\alpha} \underline{v}_{\alpha}, \alpha=i, j, e ; q_{\alpha}$ represents the charge of the species of the plasma. 


\section{DISPERSION FELATION, PHASE AND GROUP VELOCITIES}

We consider the propagation of whistler wave in the direction which makes an angle $\theta$ with the uniform static magnetic field $\underline{H}=\left(0,0, H_{0}\right)$. We make an assumption that all perturbed quantities vary as $\exp [i(\underline{k} \cdot \underline{r}-\omega t)]$. Making reference to Stix [7], and using the above equations, we obtain the general dispersion relation in the form

$$
A n^{4}-B n^{2}+C=0
$$

where $n=|\underline{n}|=\left|\frac{c k}{\omega}\right|$ is the refractive index of the wave and it is the ratio of the velocity of light to the phase velocity,

$$
\begin{gathered}
A=S \sin ^{2} \theta+P \cos ^{2} \theta \\
B=R L \sin ^{2} \theta+P S\left(1+\cos ^{2} \theta\right) \\
C=P R L, \quad S=\frac{1}{2}(R+L), \quad D=\frac{1}{2}(R-L) \\
P=1-\sum_{\alpha}\left(\omega_{p_{\alpha}}^{2} / \omega^{2}\right) \\
L=1-\sum_{i} \frac{\omega_{p_{j}}^{2}}{\omega\left(\omega-\Omega_{i}\right)}-\sum_{j} \frac{\omega_{p_{j}}^{2}}{\omega\left(\omega+\Omega_{j}\right)}-\frac{\omega_{p_{e}}^{2}}{\omega\left(\omega+\Omega_{e}\right)}, \\
R=1-\sum_{i} \frac{\omega_{p_{i}}^{2}}{\omega\left(\omega+\Omega_{i}\right)}-\sum_{j} \frac{\omega_{p_{j}}^{2}}{\omega\left(\omega-\Omega_{j}\right)}-\frac{\omega_{p_{e}}^{2}}{\omega\left(\omega-\Omega_{e}\right)}, \\
\omega_{P_{\alpha}}^{2}=\frac{4 \pi n_{0 \alpha} e^{2}}{m_{\alpha}}, \quad \Omega_{\alpha}=\left|\frac{q_{\alpha} H_{0}}{c m_{\alpha}}\right|,
\end{gathered}
$$

$q_{\alpha}=e$ for ions, $q_{\alpha}=-e$ for electrons and negative ions. All these symbols have their usual meaning.

It is noted that the dispersion relation (3.1) is modified so that the ion cyclotron whistler propagates almost along the geomagnetic field and it approximately tends to the ion cyclotron frequency. Under these conditions, the earlier derivation of group travel time receives major contributions from the left circularly polarized (LCP) wave as compared to the contributions of the right circularly polarized (RCP) wave and finally the RCP-wave's contributions have been neglected. In our present model, the contributions of both LCP and RCP waves to the group travel time are taken into account. The characteristics of variation of the phase velocity of both RCP and LCP waves against the variation of normalized wave frequency are shown in Figures 1 and 2. In this model, the plasma is assumed to contain the ions $\left(\mathrm{H}^{+}, \mathrm{He}_{\mathrm{e}}^{-}\right)$and $\left(\mathrm{H}^{+}, \mathrm{H}^{-}\right)$ in order to show the negative ion effects on the phase velocities and also to compare with the results obtained in plasmas with only positive ions (dotted lines). In moderately dense plasmas, the negative ions have significant effects on the phase velocity. Furthermore, due to addition of negative ions, it is seen that, for each 
extra negative ion, an extra resonance as well as a cut-off frequency are introduced. Consequently, there is always an extra stop-band in the RCP-wave. The resonance angle, $\theta_{r}$ of the wave is given by

$$
\tan ^{2} \theta_{r}=-(P / S)
$$

For the frequency near any ath ion cyclotron frequency, result (3.9) reduces to the form

$$
\tan ^{2} \theta=\frac{2}{x_{\alpha}} \frac{1}{m} \frac{\Delta \omega}{\Omega_{\alpha}}
$$

where $x_{\alpha}=n_{\alpha} / n_{e}, m=m_{e} / m_{\alpha}$ and $\Delta \omega=\Omega_{\alpha}-\omega$.

Now for an ion cyclotron whistler, $\left(\frac{\Delta \omega}{\Omega_{\alpha}}\right)$ can never be found less than $5 \times 10^{-3}$, at the minimum values of $\left(\frac{\Delta \omega}{\Omega_{\alpha}}\right)$. The resonance angle is approximately equal to $\frac{\pi}{2}$. But, for $\theta=\frac{\pi}{2}$, it could occur only near the equator. The satellite observations show that a very few whistlers are found near the equator as compared with the occurrence rates at. mid-latitude [8]. So it is required to impose an appropriate condition $\tan ^{2} \theta \ll \tan ^{2} \theta_{r}$, to study the whistlers at mid-latitude. Two cases are of special interest:

Case I. $\theta \ll \theta_{r}$ Using the condition, $\tan ^{2} \theta \ll \tan ^{2} \theta_{r}$, the general dispersion relation (3.1) reduces for the mid-latitude whistlers to the form

$$
n^{4} \cos ^{2} \theta-n^{2} S\left(1+\cos ^{2} \theta\right)+R L=0
$$

This gives two modes of the refractive index $n$ :

$$
n_{1,2}^{2}=\frac{S\left(1+\cos ^{2} \theta\right) \pm\left\{S^{2}\left(1-\cos ^{2} \theta\right)^{2}-4 D^{2} \cos ^{2} \theta\right\}^{\frac{1}{2}}}{2 \cos ^{2} \theta}
$$

Due to the addition of extra ions, the circularly polarized waves would have a critical frequency at which polarization reversal may occur [7]. The addition of negative ions in the plasma will introduce a critical frequency which, for equal ionic masses, is equal to the ion-cyclotron frequency. So the expression $D$ in $(3.4 c)$ must be zero at this frequency. Consequently, the refractive indices (3.12ab) assume the values

$$
n_{1}^{2} \sim \frac{1}{2}(R+L) / \cos ^{2} \theta, \quad n_{2}^{2} \sim \frac{1}{2}(R+L)
$$

where the expressions for $R(\omega)$ and $L(\omega)$ are given by

$$
R(\omega) \approx \frac{\omega_{p_{j}}^{2}}{\Omega(\Omega-\omega)}, \quad L(\omega)=\frac{\omega_{p_{j}}^{2}}{\Omega(\Omega-\omega)},
$$

$\Omega$ is the cyclotron frequency of equal ionic masses and hence $\Omega=\Omega_{i}=\Omega_{j}$. 
We first consider the refractive index involving the propagdion angle $\theta$. The mathematical analysis for the other mode follows simply by putting $\theta=0^{\circ}$. The refractive index of the first mode is then simplified to obtain

$$
n^{2} \simeq \frac{(1+x)}{2 \cos ^{2} \theta} \cdot \frac{\omega_{p_{i}}^{2}}{\Omega(\Omega-\omega)}
$$

where $x=n_{j} / n_{j}$.

The corresponding group velocity is obtained by using the expression

$$
U_{g}=\left(\frac{c}{n+\omega \frac{\partial n}{\partial \omega}}\right)\left[1+\left(\frac{1}{n} \frac{\partial n}{\partial \theta}\right)^{2}\right]^{\frac{1}{2}} \text {, }
$$

as

$$
u_{g}=U_{0} u_{0}^{\prime \prime}
$$

where

$$
\begin{array}{ll}
U_{0}^{\prime \prime \prime}=\frac{c \sqrt{\Omega}\left(\Omega_{-} \omega\right)^{3 / 2}}{\omega_{p_{i}}\left(\Omega_{-} \omega / 2\right)}, \quad U_{0}^{\prime \prime}=U_{0}^{\prime} / U_{0} \\
U_{0}=\left[\frac{1+x}{2 \cos ^{2} \theta}\right]^{\frac{1}{2}}, & U_{0}^{\prime}=\sec ^{\theta}
\end{array}
$$

Using the expression for $U_{g}$ from (3.17), we make a plot of normalized group refractive index $\mu_{g} /\left(\omega_{p_{i}} /{ }^{\Omega i}\right)_{r}$ where the group refractive index $\left.\mu_{g}=c /\left|u_{g}\right|\right)$ against the variation of the normalized frequency of the wave in figure 3 . The results are then compared with Gurnett and Shawhan's [2] result given by the dotted line for a plasma containing only the positive ion. It is seen that an appreciable variation of $\mu_{g}$ occurs with the variation of the negative ion-concentration. We also plot the ratio $U_{g} / U_{0}$ as a function of $x$ (the ratio of the concentration of negative ions to positive ions) in Figure 4.

Case II. $\theta \approx{ }_{r}$

At the equator the angle $\theta$ is nearer to the resonance angle $\theta_{r}$. So we can modify the general dispersion relation (3.1) as:

where $\quad S=\frac{1}{2}(1+x) \cdot \frac{\omega_{p_{i}}^{2}}{\omega(\Omega-\omega)}, \quad D=\frac{1}{2}(x-1) \cdot \frac{\omega_{p_{i}}^{2}}{\omega(\Omega-\omega)}$

Following earlier assumptions i.e. the wave frequency nearly equals the ion cyclotron frequency, the refractive index assumes the form:

$$
n^{2}=\frac{2(1+x) x}{(1+x)^{2}+(1-x)^{2} \cos ^{2} \theta} \cdot \frac{\omega_{p_{i}}^{2}}{\omega(\Omega-\omega)}
$$


Now expanding eauation (3.22) about $\Omega$ in terms of $\Delta \omega=\Omega_{i}-\omega$ ano reglectina the higher order terms on the parameter $\Delta \omega / s$, the refractive index reduces to the form

$$
n^{2}=\frac{2(1+x) x}{(1+x)^{2}+(1-x)^{2} \cos ^{2} \theta} \cdot \frac{\omega_{p}^{2}}{\Omega(\Omega-\omega)}
$$

and the correspording group velocity is obtained in the form

$$
\mathrm{u}_{\mathrm{g}}=\mathrm{U}_{0} \mathrm{~V}_{0}^{\prime \prime}
$$

where

$$
\begin{aligned}
& U_{0}=\frac{c \sqrt{\Omega}(\Omega-\omega)^{3 / 2}}{\omega_{p_{i}}(\Omega-\omega / 2)}, \quad V_{0}^{\prime \prime}=V_{0}^{\prime} / V_{0} \\
& V_{0}^{\prime}=\left(\frac{1}{x}\right)^{\frac{1}{2}}\left[1+\frac{a^{2} \cos ^{2} \theta \sin ^{2} \theta}{\left(b+a \cos ^{2} \theta\right)^{2}}\right]^{\frac{1}{2}}
\end{aligned}
$$

and

$$
v_{0}=\left[\frac{2}{b+a \cos ^{2} \theta}\right]^{\frac{1}{2}}, \quad a=(1-x)^{2}, \quad b=(1+x)^{2}
$$

Equation (3.25a) shows that the group velocity at the equator depends on the plasma frequency, ion-cyclotron frequency, the propagation angle $\theta$ and the concentration of negative iors. We plot the normalized group refractive index as a function of normalized wave frequency for various concentrations in Figure 5. In Figure 6, the variation of $U_{g} / U_{0}$ is plotted against the variation of the propagation angle $\theta$, for whistlers observed at the equator. We compare our results with the results obtained in the icnosphere consisting of positive ions only [results are taken from Gurnett and Shawhan [2]). It is shown that the effects of negative ions on the group velocity derived for the whistlers observed at the equator.

\section{THE GROUP TRAVEL TIME OF WHISTLERS}

The measurement of group travel time for the whistler from its scurce to an observer at the satellite is given by the line integral:

$$
t(\omega)=\int_{0}^{h} \frac{d h}{\sigma_{g}}
$$

where

$$
U_{g}=\left(\frac{c}{n+\omega \frac{\partial n}{\partial \omega}}\right)\left[1+\left(\frac{1}{n} \frac{\partial n}{\partial \theta}\right)\right]^{\frac{1}{2}}
$$

is the group velocity of the wave propagating along the path $h$. From the satellite observations on the characteristics of the whistler, the integral (4.1) has been modified. The observation reveals that the whistlers, after the formation, propagate with a rising tone along the geomagnetic field, and the frequency of the whistler approaches asymptotically the ion-cyclotron frequency in the ionosphere surrounding the satellite. Due to these observational results in the earlier chosen ionospheric model, the group travel time received a major contribution from the left circularly polarized wave. Based on the above characteristics, the travel time is modified to 
the fol?owing form:

$$
t(w)=\int_{0}^{h} \frac{d h}{U_{g L}}
$$

where $U_{\mathrm{oL}}$ is the group velocity of the LCF viave. But in our present model both the $L C P$ and $R C P$ waves have contributions to the group travel time and then $U_{g L}$ is modified tc. ${ }^{g}$. The prestnt study shows that earlier studies have been confined to an ideal model of ionosphere consisting of several species of positive ions. This model may be an idfal one in diagnosing the ionosphere, but the existence of negative ions in the icnosphere indicates that the previously chosen model becomes purely a degenerate case unless a proper account of negative ions is taken. Smith [9] has shown the effects of negative iors on the study of whistler propagation in the ionosphere.

5. THE GROUP TRAVEL TIME OF THE WHISTLERS AT MID-LATITUDE

The çroup travel time of the mid-latitude whistler is obtained in the integral form as:

$$
t(\omega)=\int_{0}^{h} \frac{d h}{U_{g}}
$$

where

$$
u_{g}=\left(\frac{2}{1+\chi}\right)^{\frac{1}{2}}{\frac{c \sqrt{\Omega}(\Omega-\omega)^{3 / 2}}{\omega_{p_{i}}(\Omega-\omega / 2)}}^{3 / 2}
$$

Equation (5.1) is the working equation to employ for the whistlers observed at mid-latitude. In order \pm 0 use this equation, we now assume that the density variation is negligibly small and the cyclotron frequency $\Omega(h)$ varies linearly with respect to $h$ and so we write

$$
\Omega(h)=\Omega(0)+h \Omega^{\prime}(0)
$$

where $\Omega(0)$ is the cyclotron frequency at the satellite and $\Omega^{\prime}(0)$ is the gradient of the cyclotron frequency to be determined from the geometry of geomagnetic field variation. The integral equation (5.1) after a simple mathematical manipulation, can be written as:

$$
t(\omega)=\frac{1}{2 c U_{0}^{\prime \prime}} \underset{\Delta \omega(0)}{\Delta \omega(h)} \frac{\left.{ }^{\omega} p_{j}(h)[\Omega)\right]^{\frac{1}{2}} d(\Delta \omega)}{\Omega^{\prime}(0)[\Delta \omega(h)]^{3 / 2}}
$$

The negligible small variation of density allows us to take the plasma frequency out of the integral and it then becomes easy to do further simplification to evaluate the integral for $t(w)$ :

$$
t(\omega)=\frac{1}{U_{0}^{\prime \prime}} t^{\prime}(\omega)
$$

where $\quad U_{0}^{\prime \prime}=\left(\frac{2}{1+\chi}\right)^{\frac{1}{2}}$ and $t^{\prime}(\omega)=\frac{{ }_{p_{i}}(0)[\Omega(0)]^{\frac{1}{2}}}{c \Omega^{\prime}(0)[\Delta \omega(0)]^{\frac{1}{2}}}$

The quantity $t(\omega)$ is the group travel time determined in our present model; we find that $t(\omega)$ is modified due to the presence of negative ions in the plasma. Equation 
(5.4) shows that the presence of negative ions introduces a correction factor $\left(\frac{1+x}{2}\right)^{\frac{1}{2}}$ to the group travel time obtained by Gurnett and Shawhan [2]. We have shown the variation of $T^{\star}=t(\omega) / t^{\prime}(\omega)$ with the variation of $x$ in Fig. 7 , which shows that the increase of the percentage of the negative ions increases the relative group travel time.

6. THE GROUP TRAVEL TIME OF WHISTLERS AT THE EQUATOR

Substituting (3.25a) into (4.3), the group travel time of the equatorial whistlers is obtained in the form

$$
t(\omega)=\int_{0}^{h} \frac{d h}{U_{0} V_{0}^{m}}
$$

where

$$
V_{0}^{\prime \prime}=\left[\frac{b^{2}+2 a b \cos ^{2} \theta+a^{2} \cos ^{2} \theta}{2 x \sqrt{b}\left(b+a \cos ^{2} \theta\right)}\right]^{\frac{1}{2}}
$$

Expression (6.1) for $t(\omega)$ can be written in the modified form:

$$
t(\omega)=\frac{\omega_{p_{j}}(0)[\Omega(0)]^{\frac{1}{2}}}{c V^{\prime \prime} \Omega^{\prime}(0)[\Delta \omega(0)]^{\frac{1}{2}}}
$$

A comparison of (6.3) with the result of Gurnett and Shawhan [2] reveals that the group travel time is modified by the factor $1 / V_{0}^{\prime \prime}$ which is a function of $\theta$ and the concentration of negative ions. Figure 8 shows a plot of $t(\omega) / t^{\prime}(\omega)$ against the concentration of the negative ions as well as with the variation of $\theta$. The variation indicates that $T^{*}$ is not much changed by the propagation angle, whereas the presence of negative ions in the ionosphere does significantly influence the variation of $T^{*}$. Equation (6.3) through dependent on the plasma frequency and cyclotron frequency is greatly influenced by the concentration of negative ions present in the ionosphere.

\section{CYCLOTRON DAMPING OF WHISTLERS AT MID-LATITUDE}

As few whistlers are observed at the equator, we consider here the cyclotron damping of the mid-latitude whistlers only. When $\omega \approx \Omega$ some protons will have a velocity component due to the thermal motion, and will experience a higher wave frequency [10]. When the wave frequency becomes equal to the cyclotron frequency of protons, then protons absorb energy by cyclotron damping. Now for a purely longitudinal wave with isotropic Maxwellian velocity distribution of ions, the dispersion relation of the LCP and RCP waves are given by

$$
L=1+\frac{\omega_{p_{i}}^{2}}{\Omega(\Omega-\omega)}+\frac{i \sqrt{\pi} \omega_{p_{i}}^{2}}{\omega / k \mid V_{i}} \exp \left(-z^{2}\right)
$$

and

$$
R=1+\frac{\omega_{p_{j}}^{2}}{\Omega(\Omega-\omega)}+\frac{i \sqrt{\pi} \omega_{p_{j}}^{2}}{\omega / k T V_{j}} \exp \left(-z^{2}\right)
$$

where $z=\frac{\Delta \omega}{k V_{i}}, T_{i}=T_{j}=$ temperature of the $i$-th and $j$-th ions, $v_{i}=v_{j}=$ thermal 
velocitu of the $i$-th and $j$-th ions.

Substituting equation (7.1) aric (7.2) in the second mode for $n$ in equation (3.13b), we obtain

$$
\frac{k^{2} c^{2}}{\omega^{2}}=1+\left(\frac{1+x}{2}\right) \frac{\omega_{p_{i}}^{2}}{\Omega(\Omega-\omega)}+\left(\frac{1+x}{2}\right) \frac{i \sqrt{\pi}}{\omega|k|} \cdot \frac{\omega_{p_{i}}^{2}}{V_{i}} \exp \left(-z^{2}\right)
$$

For the temporal damping phenomena, we assume $k$ is real, and $\omega=\omega_{r}+i \omega_{i}$ with $\left|\omega_{r}\right| \gg\left|\omega_{j}\right|$. Now, following Stix [7?, Singh et al [11] and Das and Sur [12], we find

$$
\omega_{i}=-\left(\frac{2}{1+\chi}\right)^{\frac{1}{2}} \frac{\sqrt{\pi} \Omega(0)^{\frac{1}{2}}[\Delta \omega(0)]^{3} c}{2 V_{i}{ }^{\omega} p_{i}}(0) \exp [-n(0)]
$$

where

$$
n(h)=\left(\frac{2}{1+x}\right) \frac{c^{2}[\Delta \omega(h)]^{3}}{\Omega(0) \omega_{p_{i}}(0)^{2} v_{i}^{2}}
$$

From the expression (5.4) for group trave! time of mid-latitude whistlers, we obtain

$$
\Delta \omega(0)=\left(\frac{1+x}{2}\right) \frac{\omega_{p_{i}}^{2}(0 ; \Omega(0)}{c^{2}\left[\Omega^{\prime}(0)\right]^{2}} \cdot \frac{1}{t^{2}}
$$

Substituting the value of $\Delta \omega(0)$ from the equation (7.6) in the equation (7.4) the temporal damping parameter, $\omega_{i}$ is given by

$$
\omega_{i}=G t^{-3} \exp \left[-\gamma t^{-6}\right]
$$

where $\quad G=-\left(\frac{1+x}{2}\right) \frac{\sqrt{\pi}}{2 V_{i}}\left[\frac{\Omega(0) \omega p_{i}(0)}{c}\right]^{2} \cdot \frac{1}{\Omega^{\prime}(0)^{3}}$

$$
Y=\left(\frac{1+x}{2}\right)^{2}\left[\frac{\omega_{p_{i}}(0)}{c}\right]^{4}\left[\frac{\Omega(0)}{V_{i}}\right]^{2} \cdot \frac{1}{\left[\Omega^{\prime}(0)\right]^{6}}
$$

The velocity distribution function $F(v)$ is related to $\omega_{i}$ by the following relation given in [13]:

$$
\omega_{i}=-\frac{2 \pi^{2} c[\Delta \omega(0)]^{5 / 2}}{\omega_{p_{i}}(0) \Omega(0)^{\frac{1}{2}}} F(v)
$$
form

Substituting $\omega_{i}$ from equation (7.7) in equation (7.10), $F(v)$ is obtained in the

where

$$
\begin{gathered}
F(v)=M \exp \left[-Y t^{-6}\right] \\
M=\left(\frac{2}{1+\chi}\right)^{\frac{1}{2}} \cdot \frac{1}{4 \pi^{3 / 2} V_{i}} \cdot \frac{\Omega(0)}{\Delta \omega(0)} .
\end{gathered}
$$


When $w$ is real and $k=k_{r}+i k_{j}$ with $\left|k_{r}\right| \gg\left|k_{j}\right|$, the separation of real and imaginary parts of the expression (7.3) yields:

$$
k_{r}=\left(\frac{1+\chi}{2}\right)^{\frac{1}{2}} \frac{\omega_{p_{i}} \Omega^{\frac{1}{2}}}{c[\Delta \omega(0)]^{\frac{1}{2}}}
$$

and

$$
k_{i}=\frac{\sqrt{\pi} \Delta \omega}{2 V_{i}} \exp [-n(h)]
$$

The corresponding attenuation factor $\beta$ is obtained by integrating (7.14) over the propagation path and has the form

$$
\begin{aligned}
B & =2 \int_{0}^{h} k_{i} d h \\
& =\left(\frac{1+x}{2}\right) \cdot \frac{\sqrt{\pi} v_{i} \Omega(0) \omega_{p_{j}}(0)^{2}}{2 c^{2} \Omega^{\prime}(0) \Delta \omega(0)} \exp [-n(0)]
\end{aligned}
$$

where $\Delta \omega(0) \ll \Delta \omega(h)$ i.e. $n(0) \ll n(h)$. Using $\Delta \omega(0)$ from the equation (7.6) in the expression (7.15), the value of $\beta$ can be expressed as

$$
B=G^{\prime} t^{2} \exp \left[-Y t^{-6}\right]
$$

where

$$
G^{\prime}=\frac{\sqrt{\pi} V_{i} \Omega^{\prime}(0)}{3}
$$

In the absence of damping, we follow Gurnett and Brice [10], Das and Sur [12] to obtain

$$
\tilde{p} \sim\left|\frac{d(\Delta \omega)}{d t}\right|
$$

where $\tilde{P}$ is the poynting flux.

But, for a constant poynting flux, $\frac{\left|B_{1}\right|}{\left|E_{1}\right|}$ is directly proportional to the refractive index, that is,

$$
\frac{\left|B_{1}\right|}{\left|E_{1}\right|} \propto n \text { a }(\Delta \omega)^{-\frac{1}{2}}
$$

and from (5.4), it turns out that

$$
\left|B_{1}\right|\left|E_{1}\right| \propto \frac{d}{d t}(\Delta \omega) \propto(\Delta \omega)^{3 / 2}
$$

Combining (7.19) and (7.20) and using $\Delta \omega(0)$ from (7.6), the relative amplitude of the magnetic field is obtained as

$$
\left|B_{1}\right| \propto(\Delta \omega)^{\frac{1}{2}} \propto t^{-1}
$$


If the cyclotron damping is included, then

$$
\begin{aligned}
B_{1} & \alpha \mathrm{e}^{-\beta} \\
& \alpha \exp \left[-G^{\prime} t^{2} \exp \left(-Y t^{-6}\right)\right]
\end{aligned}
$$

Combining (7.21) and (7.22), we find that

$$
B_{1} \propto \frac{1}{t} \exp \left[-G^{\prime} t^{2} \exp \left(-Y t^{-6}\right)\right]
$$

\section{DISCUSSIONS AND CONCLUDING REMARKS}

This analysis reveals that the estimation of cyclotron damping along with the velocity distribution as well as the amplitude of the magnetic field in both cases depend on the group travel time. To show that effects of negative ions on the damping of whistlers we consider here the proton whistler data of Gurnett and Brice [10] for which $\Delta \omega(0), \Omega_{0}(0)$ and $\eta\left(H^{+}\right)$are 3.7 C.P.S., 410.3 C.P.S. and $12 . x \times 10^{3} /$ C.C. respectively. The plots are considered for three different temperatures $600^{\circ} \mathrm{K}, 800^{\circ} \mathrm{K}$ and $1000^{\circ} \mathrm{K}$ with a fixed value of $x=0.1$.

The variation of $\omega_{i}$ against the variation of $t(\omega)$ is shown in Fig. 9. It is clear from Fig. 9 that the temporal damping starts 3 secs. delay from the starting of the whistler, and there is a steep rise of the damping at $t=3.5 \mathrm{sec}$. The plot for three different temperatures, shows the effective role of temperature on $\omega_{j}$. The maximum value of $\omega_{i}$ is independent of temperature nearly equal to 156 C.P.S. at $t \sim 5$ sec.

Variation of the velocity distribution function $F(v)$ with the variation of $t(\omega)$ is shown in Fig. 10. At $t=5 \mathrm{sec}, F(v)$ reaches a constant value approximately for different temperatures. The increase of the attenuation ${ }_{\beta}(\beta)$ with both group travel time and temperature is shown in Fig. 11.

The relative wave amplitude of the magnetic field is sensitive due to the negative ion effects on the whistler (Fig. 12). Fig. 12 shows a comparison of the effect of the negative ion (solid line) with the result obtained by Gurnett and Brice [10] (the dotted line).

The present analysis of the propagation of whistlers in the ionosphere reveals the effect of negative ions on the estimation of the group travel time as well as cyclotron damping of the ion-cyclotron whistlers. The plot of $(\Delta \omega)^{-\frac{1}{2}}$ against the group travel time $t(\omega)$ provides an estimation of the ion-cyclotron frequency and the relative concentration of ions. The velocity distribution, total attenuation and the induced magnetic field have been calculated from the temporal as well as spatial cyclotron damping. The results of this analysis clearly show that negative ions in the ionosphere have an effect on the technique employed to estimate the ionospheric parameters observed both at the mid-latitude as well as at the equator. Moreover, the present model indicates that the negative ioris should be taken into account in the ionosphere. Otherwise, a discrepancy on the results will occur. 
Finally, the present study also needs a further extension to include the following effects:

(i) The thermal effects in the ionospheric plasma seem to have some important. contribution to the propagation of whistlers. So a study of thermal effects on the whistler in the presence of negative ions will be made in a subsequent paper.

(ii) The stream velocity of the plasma and newly born ion effects in the ionosphere may also be taken into account. This problem may be studied in the future.

\section{POSSIBLE APPLICATIONS}

(i) Group Travel Time of Whistlers

Results (5.4) and (6.3) for the group travel time represent the working equations for determination of the physical parameters in the ionosphere. They can be used as diagnostic tools for ionospheric studies. For such a use, it is necessary to plot $t(\omega)$ against $(\Delta \omega)^{-\frac{1}{2}}$ for several cyclotron frequencies for a set of observations. As an example, we select an arbitrary ion-cyclotron frequency, $\Omega_{1}$ (say) and make a plot of $(\Delta \omega)^{-\frac{1}{2}}$ against $t(\omega)$. If the frequency is small compared to the actual frequency, an upward concave line will be observed as shown in Figure 13 with the mark $I$. In this case, we need to increase the cyclotron frequency $s_{i}$ and repeat the plot $(\Delta \Omega)^{-\frac{1}{2}}$ against $t(\omega)$ to minimize the concavity. Similarly, if we have chosen high frequency then one will observe a downward concave line (marked by II) and leads to lower the arbitrary cyclotron frequency. We repeat the plots with several chosen ion-cyclotron frequencies until one should get the best straight line; the cyclotron frequency for which we should get the straight line is the actual ion-cyclotron frequency.

Now the slope of the straight line obtained for mid-latitude and equatorial whistlers for the actual ion-cyclotron frequency may be used to determine the relative concentration of ions, since $\Omega^{\prime}(0)$ can be obtained from the geometry of the geomagnetic field variations and $\omega_{p_{j}}(0)$ may be used from the ionospheric data. Thus the ion-cyclotron frequency and the relative concentration of ions may be obtained through the estimation of the group travel time.

(ii) Heating of the Ionosphere

It is known that absorption of energy through attenuation of the waves is one of the sources of changing the physical properties of the medium. The ionosphere as well as the atmospheres of other astrophysical bodies are heated by the energy gained from the attenuated waves. Barnes [14] showed that hydromagnetic waves become damped even in collisionless plasma and the solar corona may be heated by this mechanism. Bandyopadhyaya [15] pointed out that the rotational astrophysical objects plays an important role in damping of the MHD waves and it causes heating of the rotating stars. There are several theories to explain the heating phenomena of earth's ionosphere [16-20], but no theory has been proved to be unique. Recently, Murtaza and Shukla [6] pointed out that the Landau damping of the lower hybrid waves can cause the electron heating, whereas the ion-cyclotron waves can heat the ions preferentially, i.e. both the plasma species could gain energy at the expense of the whistler wave. Our present analysis for the damping of whistler waves due to nonlinear interaction with the plasma particles in the ionosphere gives a new theoretical prediction for the 
heating of the lower ionosphere. In fact, the energy absorbed in the ionosphere due to attenuation of whistlers is not large because the energy of whistlers is not large in general. But under some suitable physical conditions, particularly in resonant cases, the nonlinear absorption of energy due to damping of whistlers may be significant and it would act as one of the important sources of heating for the earth's ionosphere. The heating rate by the process of nonlinear attenuation of whistlers would be estimated in our subsequent work. It is to be mentioned here that besides heating of the medium the damping of whistlers has also other applications. The velocity distributions and the induced electric and magnetic fields may be estimated from the damping rate of whistlers [11-12]. The detailed study and numerical estimations of these parameters would be made in the future.

Acknowledgement. This work was partially supported by the University of Central Florida.

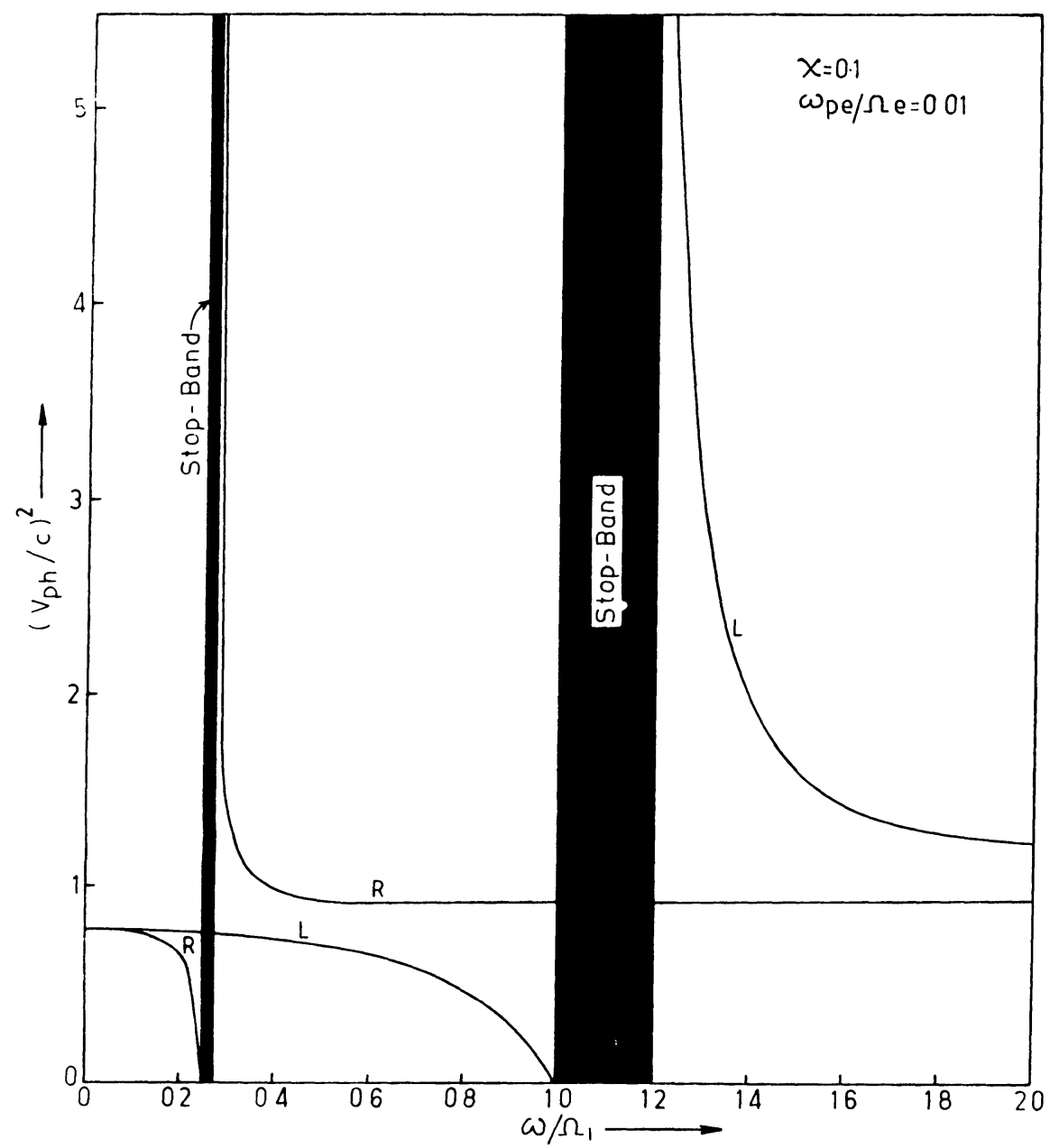

Fig. 1 Variation of phase velocity square as a function of normalized wave frequency, $\omega / \Omega$, for $\mathrm{H}^{+}, \mathrm{He}^{-}$plasmas. 


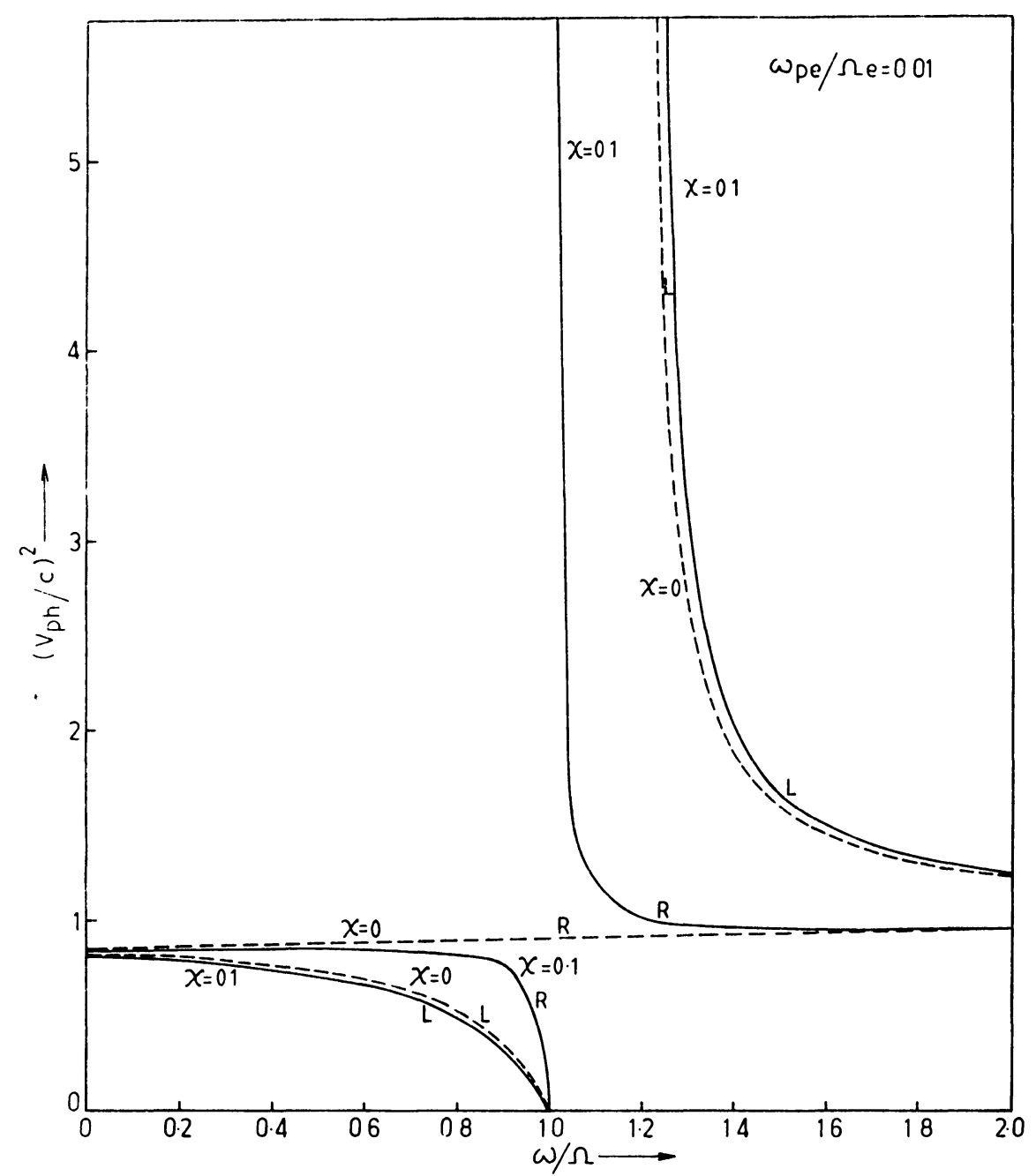

Fig. 2 Variation of phase velocity square as a function of normalized wave frequency, $\omega / \Omega$ for plasmas with (i) $\mathrm{H}^{+}, \mathrm{H}^{-}$ions and $(i i) \mathrm{H}^{+}$ions. 


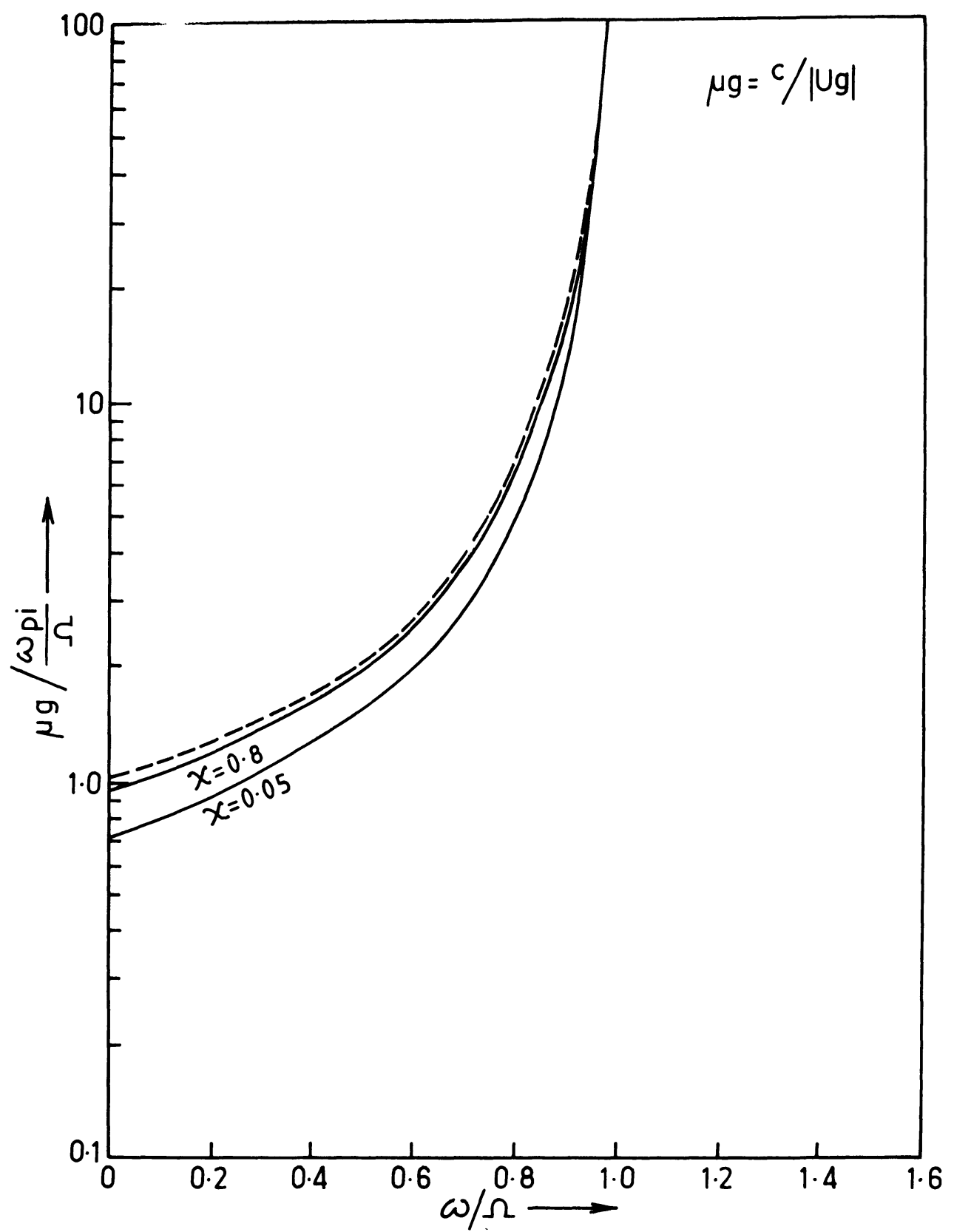

Fig. 3 Variation of normalized group refractive index, $\mu_{g} /\left(\omega_{p_{j}} / \Omega\right)$, for the whistlers observed at the midlatitude with the variation of normalized wave frequency, $\omega / \Omega$, for different values of $\chi$ the rate of concentration of negative ions to the positive ions. 


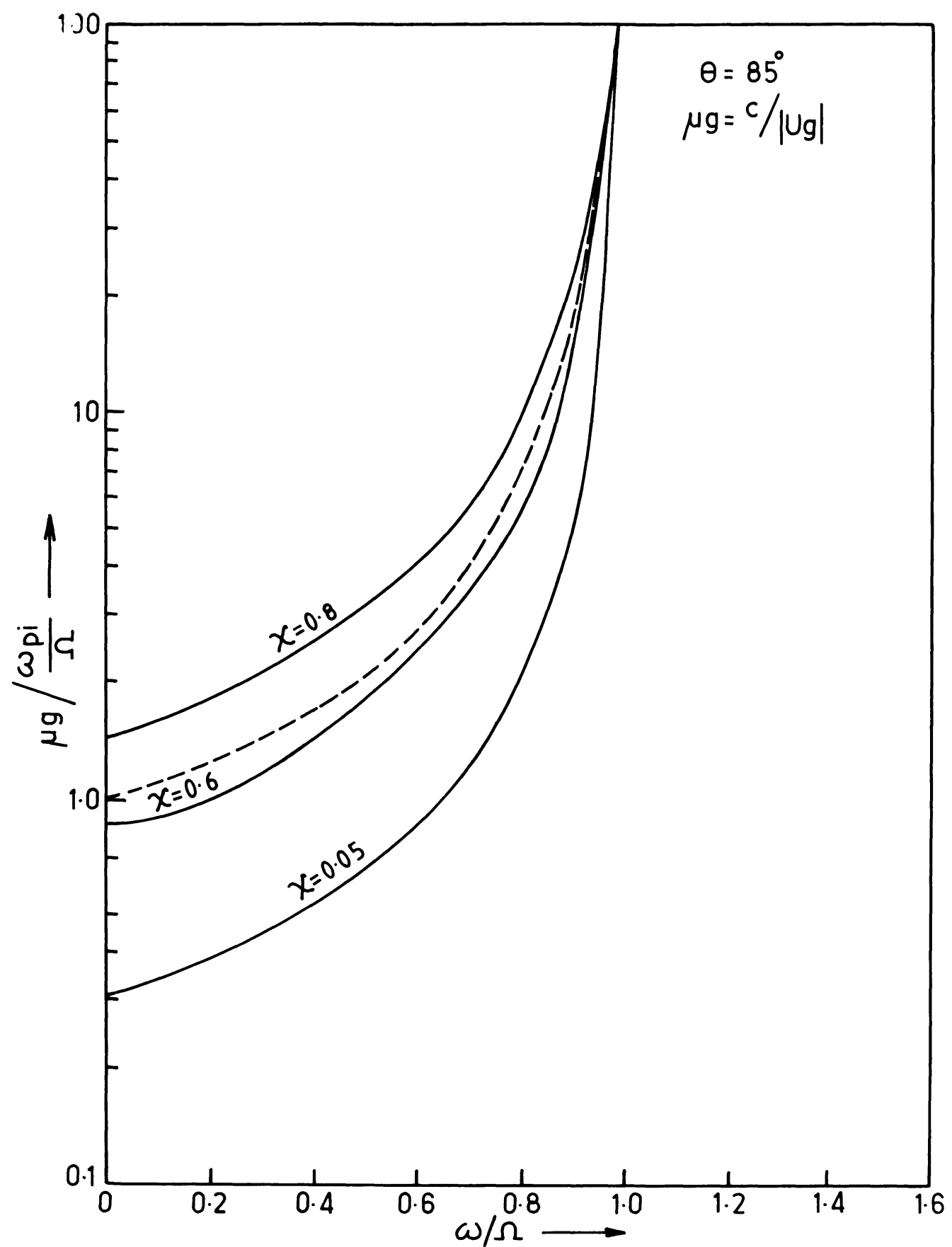

Fig. 5 Variation of normalized group refractive index, $\mu_{g} /\left(\omega_{p_{i}} / \Omega\right)$, for the whistlers observed at the equator with the variation of normalized wave frequency, $\omega / \Omega$ for different values of $x$. 


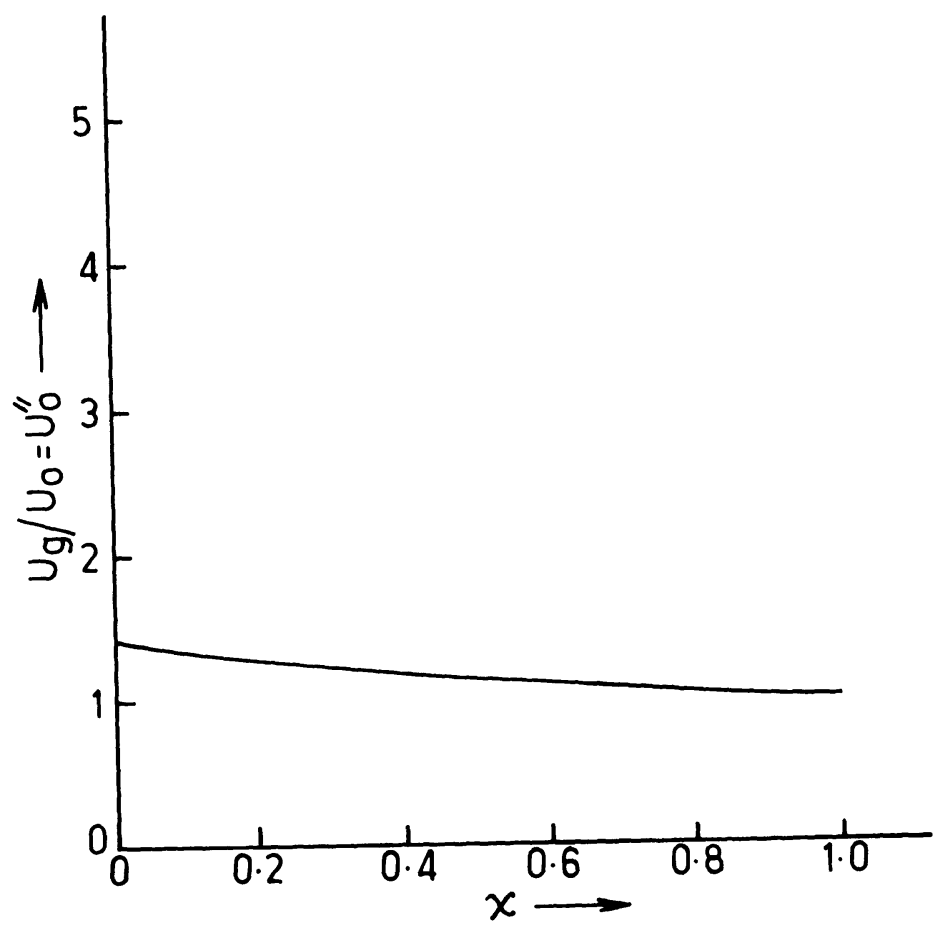

Fig. 4 Plot of $U_{g} / U_{0}$ (for midlatitude) against $x$, the ratio of concentration of negative ions to the positive ions.

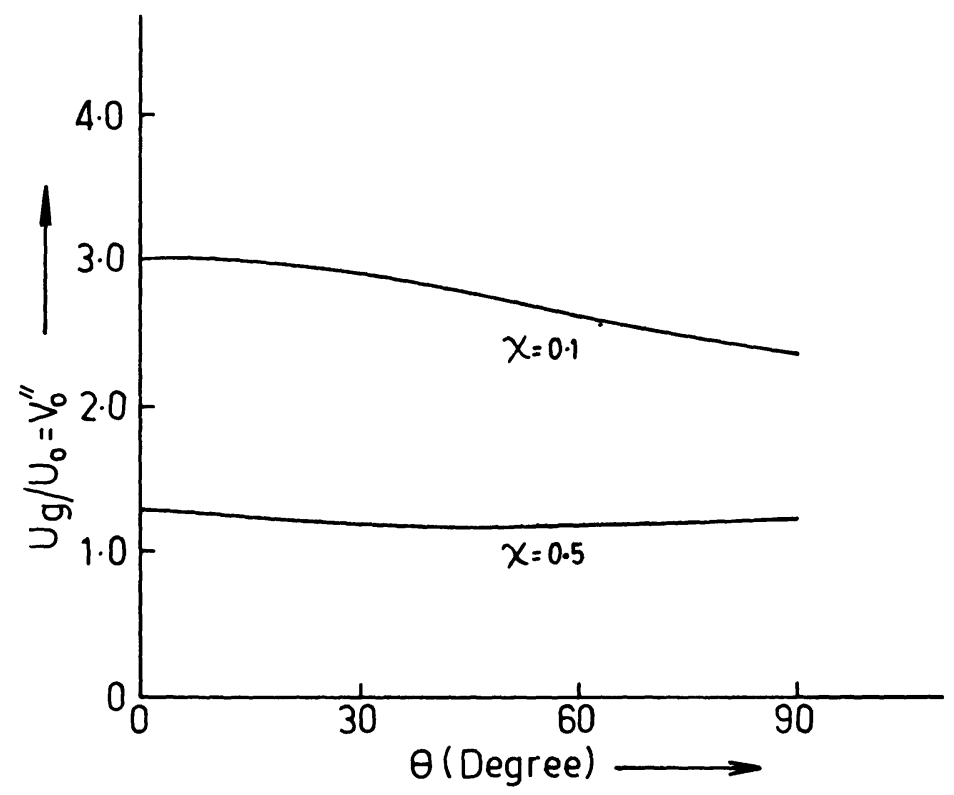

Fig. 6 Plot of $U_{g} / U_{0}$ against $\theta$ for different values of negative ion concentration at the equator. 


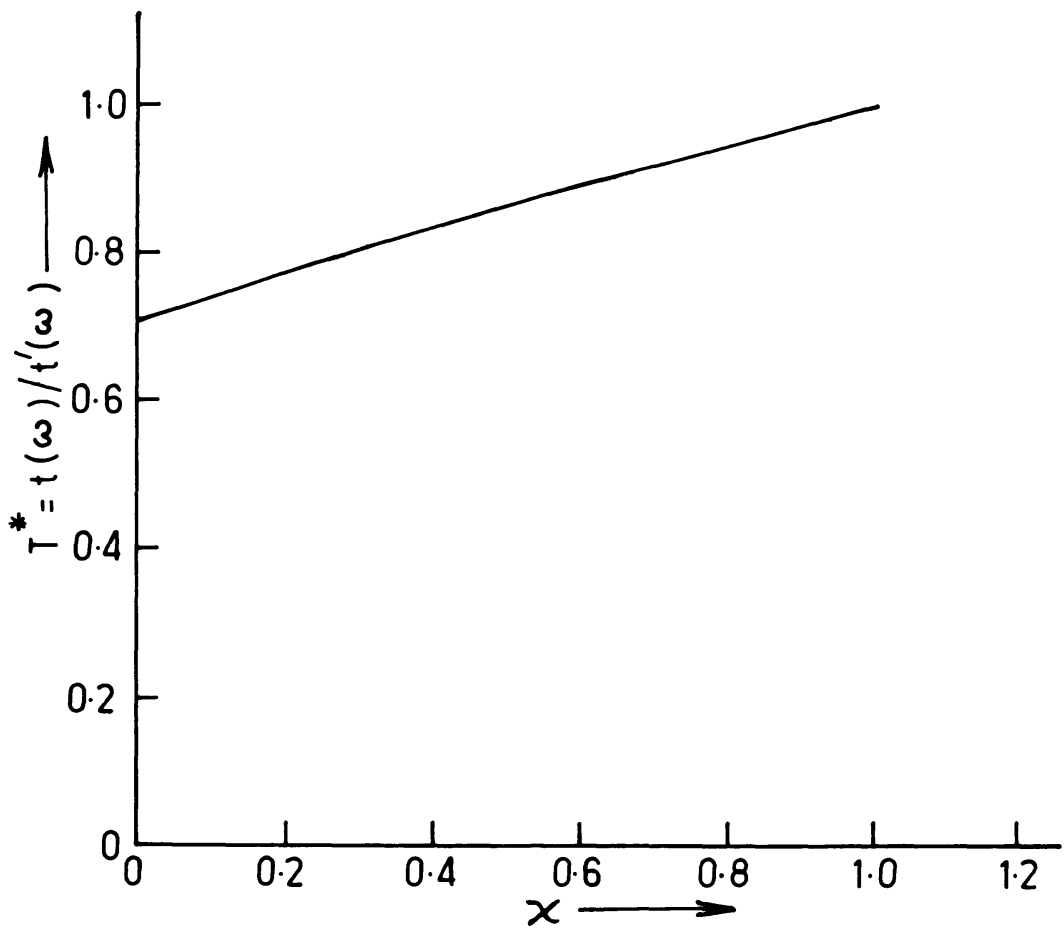

Fig. 7 Plot of $t(\omega) / t^{\prime}(\omega)$ against the ratio of the concentration of negative ions to the positive ions for the midlatitude.

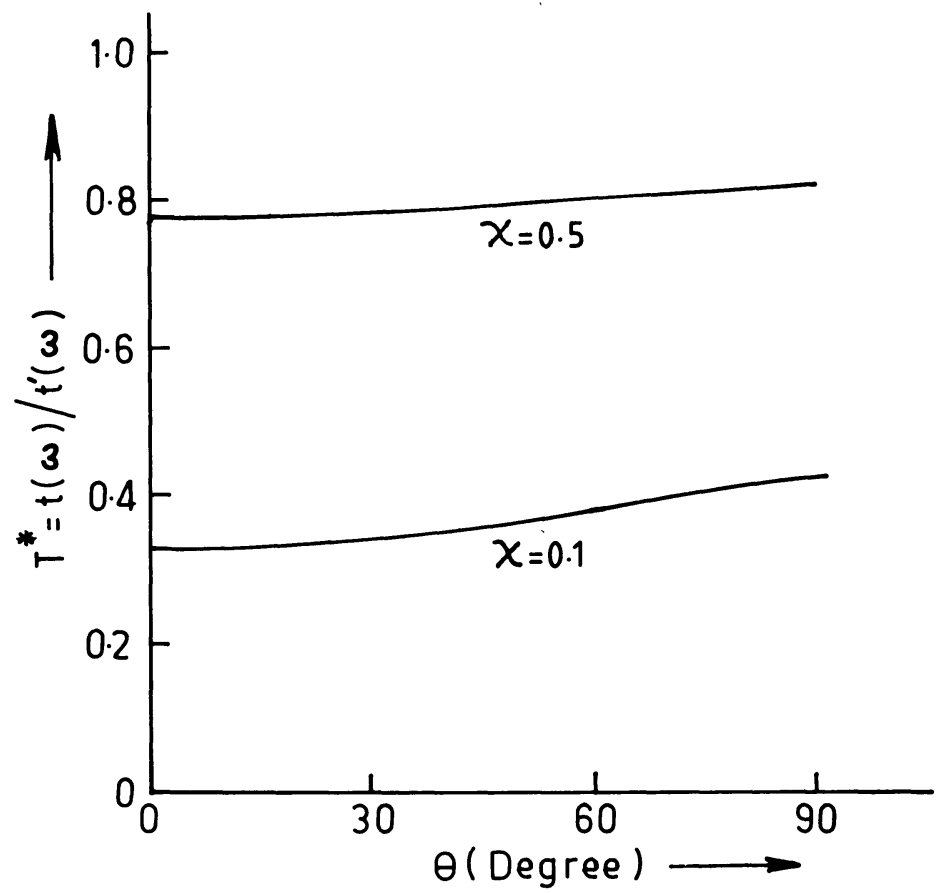

Fig. 8 Plot of $t(\omega) / t^{\prime}(\omega)$ against the concentartion of negative ions as well as with the variation of $\theta$ for the whistlers at the equator. 


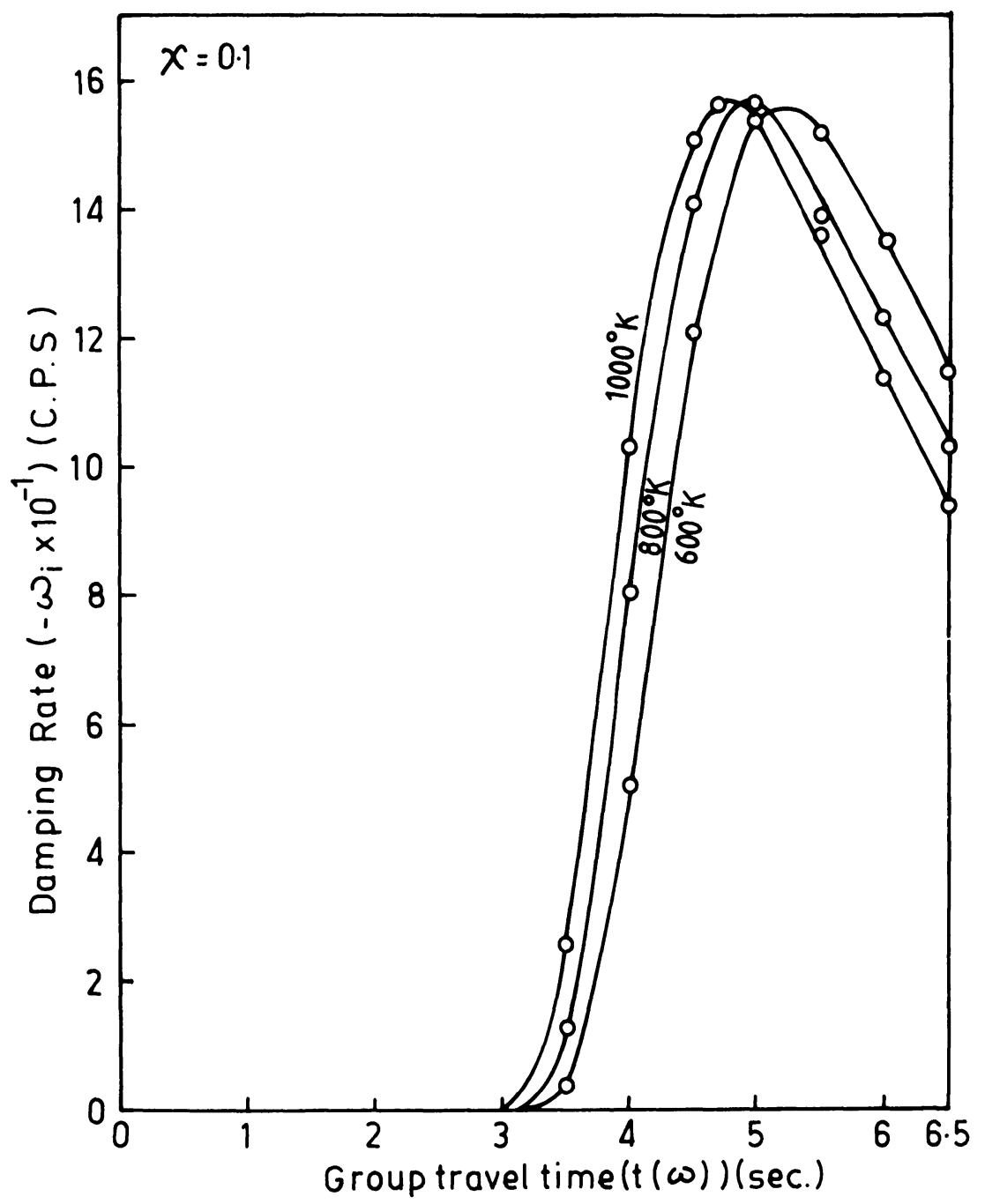

Fig. 9 Variation of temporal damping rate with the group travel time. 


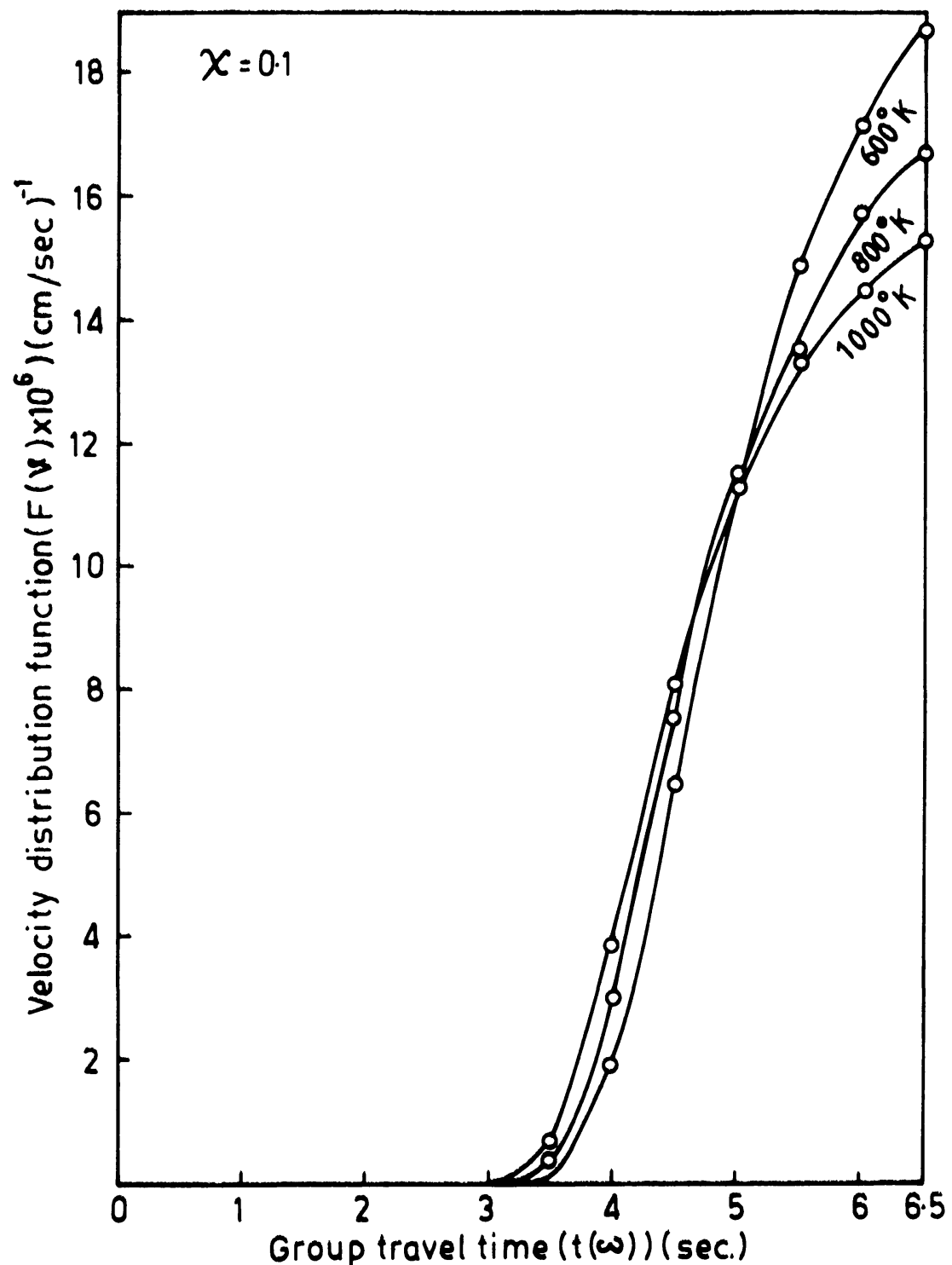

Fig. 10 Variation of velocity distribution function $F(v)$ with the group travel time $t(\omega)$. 


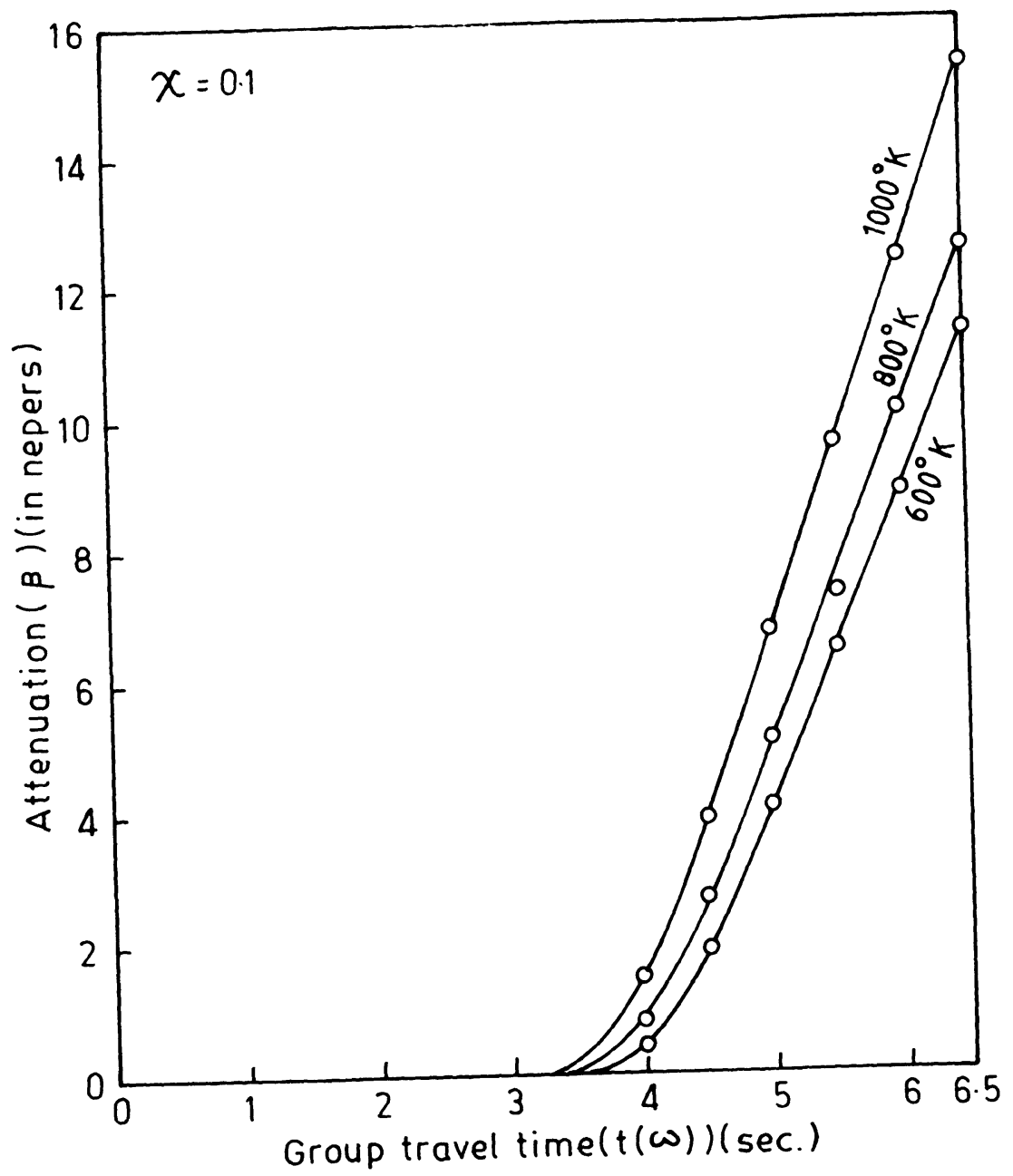

Fig. 11 Variation of total attenuation $B$ with respect to the group travel time, $t(\omega)$. 


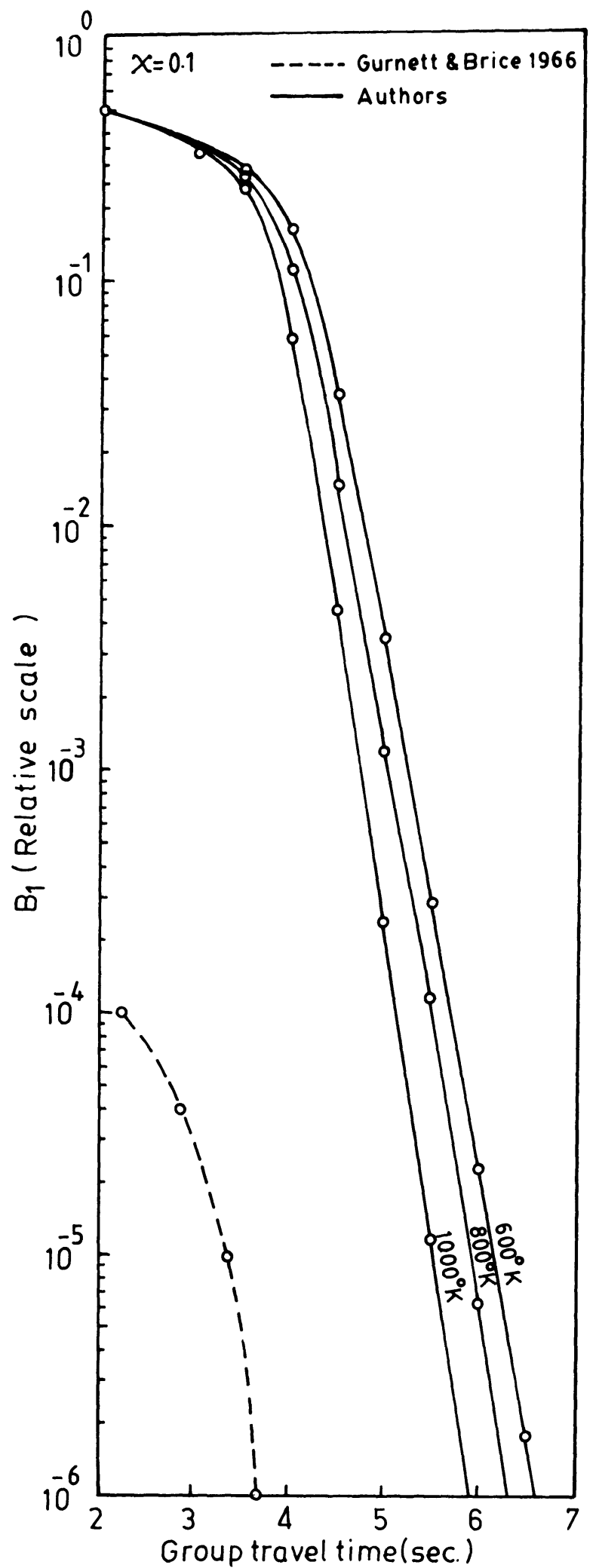

Fig. 12 Variation of the magnative field $B_{1}$, with respect to the group travel time $t(\omega)$ and negative ion concentration. 


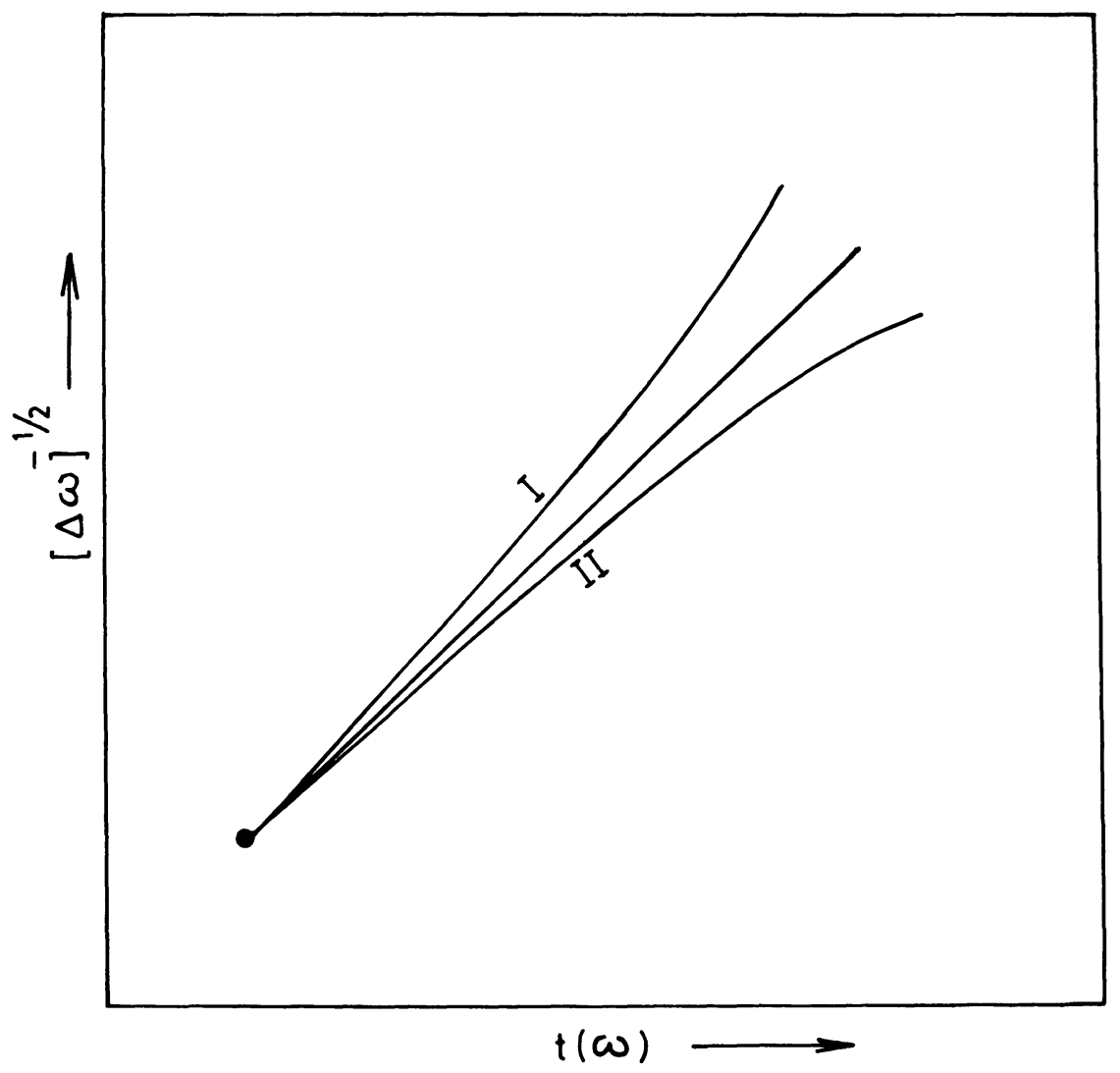

Fig. 13 A plot of $(\Delta \omega)^{-1 / 2}$ versus $t(\omega)$ for the determination of ion-cyclotron frequency for the whistlers both at the equator and midlatitude.

\section{REFERENCES}

[1] Gurnett, D. A., Shawhan, S. D., Brice, N. M. and Smith, R. L., Ion cyclotron whistlers, J. Geophys. Res. 70, (1965), 1665-1688.

[2] Gurnett, D. A. and Shawhan, S. D., Determination of hydrogen ion concentration, electron density and proton gyrofrequency from the dispersion of proton whistlers, J. Geophys. Res. 71 (1966), 741-754.

[3] Das, G. C. and Uberoi, C., Crossover frequencies in multicomponent plasmas with negative ions, J. Geophys. Res. 77 (1972), 5597-5601.

[4] Das, G. C., Ions acoustic solitary waves in multicomponent plasmas with negative ions, IEEE PS-3 (1975), 168-173. 
[5] Arno?d, F. and Krankowsky, D., Mid-latitude lower ionosphere structure and composition measurements during winter, J. Atmos. Terr. Phys. 41 (1979) 1127-1140.

[6] Murtaza, G. and Shukla, P. K., Decay of a whistler, Phys. Lett. A (Netherlands) 104A $(1984), 382-384$.

[7] Stix, T. H., The theory of plasma waves, McGraw Hill, N.Y. (1962).

[8] Shawhan, S. D., Experimental observation of proton whistlers from the Injun 3 VLF data, J. Geophys. Res. 71 (1966), 29-45.

[9] Smith, J., Negative ion-effects on whistler mode propagation, J. Geophys. Res. 70 (1965), 53-59.

[10] Gurnett, D. A. and Brice, N. M., Ion temperature in the ionosphere obtained from cyclotron damping of proton whistlers, J. Geophys. Res. 71 (1966), 3639-3652.

[1]] Singh, S. N., Tiwari, S. and Tolpadi, S. K., Characteristics of electron ion whistiers and their appi ication to ionospheric probing, J. Geophys. Res. 81 (1976), 1327-1330.

[12] Das, G. C. and Sur, A. K., A Comparison study between the newly born ion effect and thermal effect on the whistlers in the ionosphere, Astrophys. Space Sci. 127 (1986), 249-263.

[13] Lucas, C. and Brice, N., Irregularities in proton density deduced from cyclotron damping of proton whistlers, I. Geophys. Res. 76 (1971), 92-99.

[14] Barnes, A. I., Collisionless heating of the solar wind plasnlas II, Application of the theory of plasma heating by hydromagnetic waves, Ap. J. 155 (1969), 311-321.

[15] Bandyopadhyaya, R., Heating of astrophysical plasma due to rotation, Czech. J. Phys. B22 (1972), 1199-1201.

[16] Ganguli, G., Palmadesso, P., Lee, Y. C., A new mechanism for excitation of electrostatic ion cyclotron waves and associated perpendicular ion heating, Geophys. Res. Lett. 12 (1985), 643-646.

[17] Gross, S. H., Unusual heating events at about $250 \mathrm{~km}$ altitude at very low latitudes seen by AEE, J. Atmosph. Terr. Phys. 47 (1985), 941-944.

[18] Field, E. C., Warber, C. R., Ionospheric heating with obliquely incident waves, Geophys. Res. Lett. 12 (1985), 761-763.

[19] Lathuilere, C., GIangeaud, F., Zhao, Z. Y., Ionospheric ion heating by ULF Pc5 magnetic pulsations, J. Geophys. Res. 91 (1986), 1619-1626.

[20] Primdan1, F., Polar Ionospheric E-region plasma wave subilization and electron heating by wave-induced enhancement of the electron collision frequency, Phys. Scr. 33 (1986), 187-191.

List of authors by name:

\author{
A. K. Sur \\ Plasma Physics Group \\ Department of Mathematics \\ Jadavpur University \\ Calcutta - 70032 \\ INDIA \\ B. Chakraborty \\ Plasma Physics Group \\ Department of Mathematics \\ Jadavpur University \\ Calcutta - 700032 \\ INDIA
}

G. C. Das ${ }^{\star}$

Department of Mathematics

Manipur University

Imphal, Manipur

INDIA

S. N. Paul ${ }^{\star \star}$

Serampore Girls' College

P.0. - Serampore

Dist. Hooghly

West Bengal

Pin - 712201, INDIA 


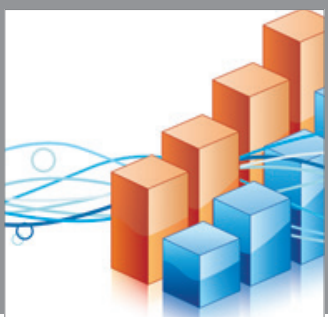

Advances in

Operations Research

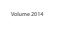

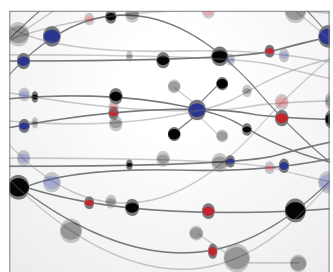

\section{The Scientific} World Journal
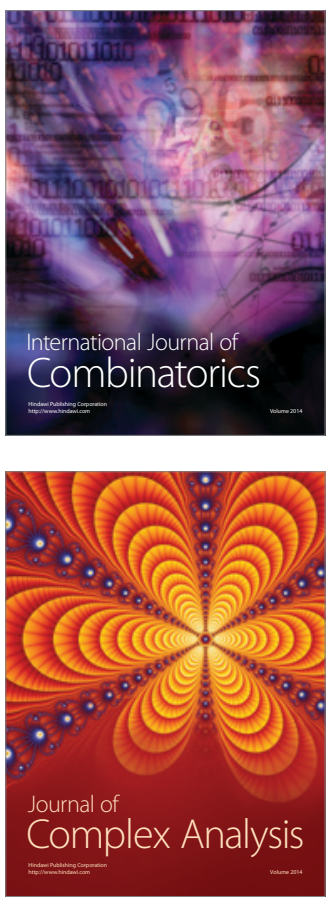

International Journal of

Mathematics and

Mathematical

Sciences
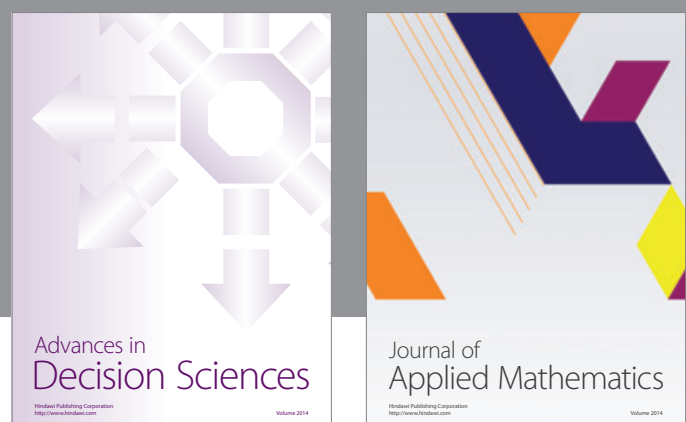

Journal of

Applied Mathematics
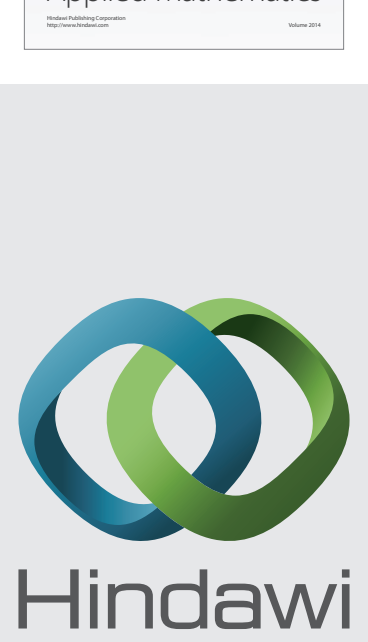

Submit your manuscripts at http://www.hindawi.com
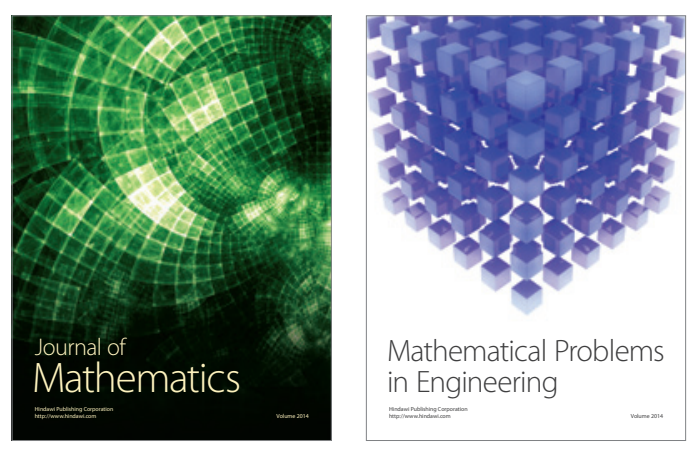

Mathematical Problems in Engineering
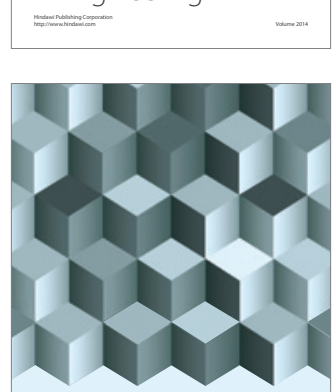

Journal of

Function Spaces
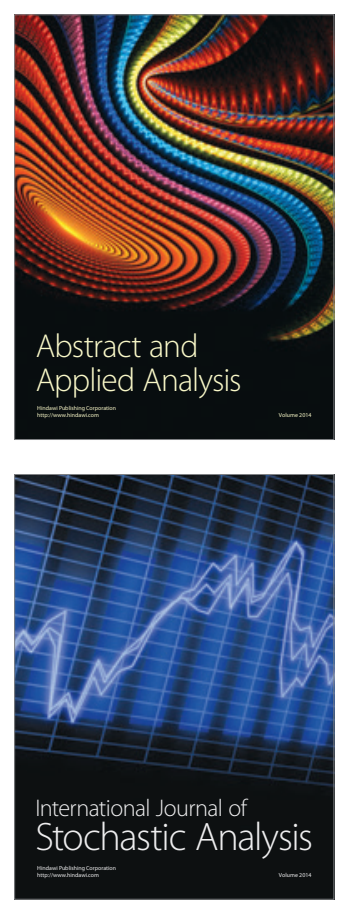

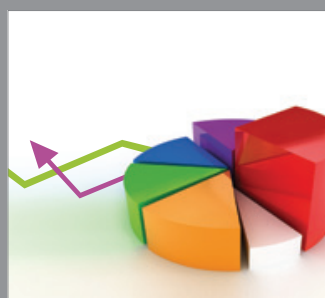

ournal of

Probability and Statistics

Promensencen
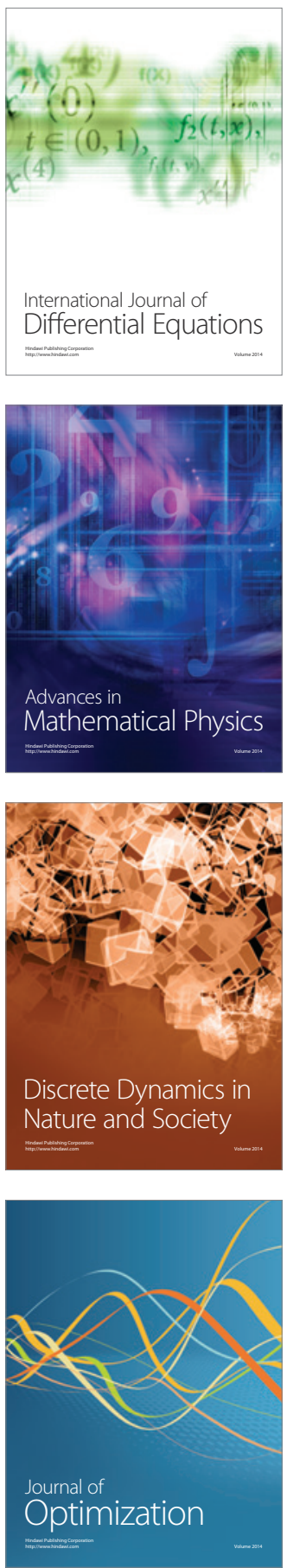